\title{
Influence of resistance to motion of railway vehicles on the longitudinal trains dynamics
}

\author{
Camil Crăciun* and Cătălin Cruceanu \\ University Politehnica of Bucharest, Department of Railway Vehicles, 313 Splaiul Independentei, \\ Romania
}

\begin{abstract}
Longitudinal dynamics of trains is a subject that generates discussions and views on the parameters that interfere and influence both the size of the forces and their distribution in the train body. The paper is a study to determine the influence of resistances to motion on the longitudinal dynamic forces that develop in the body of the train in the braking process. For this, a train study model of ten identical vehicles, to which the locomotive may or may not be attached, is adopted. Initially, the simulation program for the non-locomotive model is run in two variants: with and without introducing additional resistances to motion, followed by the same simulations but with the locomotive introduced and a wagon removed, thus the number of vehicles remains the same for all the cases presented.
\end{abstract}

\section{Introduction}

In order to understand how longitudinal dynamic forces occur and how they influence the dynamics of the trains in the braking regime, computing programs or simulations have been developed since the 1960s, worldwide, to highlight the value of the longitudinal forces, their distribution in the train body as well as the impact on the vehicle integrity or safety in service [1].

Studies of researchers such as: Cole [2], Belforte, Cheli [3], Nasr and Mohamndi [4], Pugi, Fioravanti and Ridi [5], show that longitudinal dynamic forces depend on parameters such as: operating conditions for vehicles, mass distribution in the train body, constructional and functional parameters of buffer and draw-gear devices, length of general pipeline, etc. Therefore, a study on the appreciation of longitudinal dynamic forces that takes into account all the parameters that influence them is very difficult to elaborate.

In the case of the classic braking system, because of the air compressibility and train length, there will always be a time gap between the first vehicle response and the rear vehicle response. Correspondingly, the propagation velocity of the braking wave will lead to the successive entry into action of distributors in the train body, so that, while the front vehicles are braked, those positioned at the end of the train have not developed the braking force and will collide the braked vehicles, leading to dynamic longitudinal phenomena. This can affect the travellers comfort, the integrity of the goods transported and, sometimes,

\footnotetext{
*Corresponding author: craciun_camil@yahoo.com
} 
even the traffic safety. The modelling of the braking system in order to be used in various simulation programs is presented by Pugi, Malavezzi and others [6, 7].

Jinghui Wang [8] develops a simulation model of longitudinal dynamic forces that can be implemented in a simulation program but does not depend on many mechanical parameters that complicate and make difficult the running of programs and the interpretation of results. Such a program can be used to highlight acceleration or deceleration values when the trains are in the traction or braking modes.

The evaluation of the forces that occur between the component vehicles of a train is done by means of mathematical models adapted to each constructive type of buffer and draw-gear devices. Nowadays, these devices are worldwide classified into two main categories: lateral buffers, traction devices and hand couplings (screw and traction hook coupling) and automatic couplings. A famous study that focuses on the work of all researchers on modeling buffer and draw-gear devices is performed by Qing Wu, Colin Cole et al. [9]. In this paper the authors show how different these devices are in the world, the researchers' concerns and the importance of evaluating the longitudinal forces, but also the complex or simple mathematical models used in these programs.

The present paper represents a continuation of the authors' activity in this field, see for instance $[10,11]$. Our mai target is to emphasize the effects of the introduction into the simulation programs of the resistances to motion of the train components in full braking process. Thus, in the paper we present the results obtained with and without resistances to motion, for trains composed of identical vehicles - the frame type, respectively of locomotive and identical wagons.

\section{Modelling the buffer and draw-gear devices}

The collision devices used on railway vehicles aim to protect the structure of the vehicles, to diminish the forces that occur between them during braking and to dissipate a part of the energy during collisions. Their characteristics and design type influence the development and/or diminution of longitudinal dynamic reactions with profound implications on the rolling stability of vehicles. Also, in order to study the longitudinal dynamics of trains in brake state equipped with screw-hook hand coupling, it is also necessary to know the elastic features of these devices.

In order to mathematically model the evolution of the force in the collision device, we considered the characteristic diagram of the buffer that equips the hauled passenger coach, built in Romania, whose elastic and damping element is of the RINGFEDER type (with metal rings), diagram used and presented by authors in works $[12,13]$. Considering ke a constant that depends on the elasticity of the elements within the buffer and kf a constant that depends on the friction between the metal rings within it, the force in the buffer can be evaluated using the formula [14]:

$$
F_{i}\left(x_{i}, \dot{x}_{i}\right)=\frac{\left(1-\operatorname{sgn} x_{i}\right)\left(k_{e} x_{i}+k_{f}\left|x_{i}\right| \tanh \left(u \cdot \dot{x}_{i}\right)\right)+\left(1+\operatorname{sgn} x_{i}\right)\left(k_{e c} x_{i}+k_{f c}\left|x_{i}\right| \tanh \left(u \cdot \dot{x}_{i}\right)\right)}{2}
$$

Where $\mathrm{x}$ represents the stroke of the collision device, and $\dot{x}$ the relative speed at the level of the collision device.

\section{Mechanical model for the train}

The design of the simulation program regarding longitudinal forces in the train body in braking mode is based on a general train pattern (Figure 1) of the type of a damped elastic 
system consisting of $n$ individual rigid masses $m_{i}$ - the vehicles being connected by elements having elastic $k_{i}$ and damping $c_{i}$ characteristics, well defined, corresponding to buffer and draw-gear devices [12, 14].

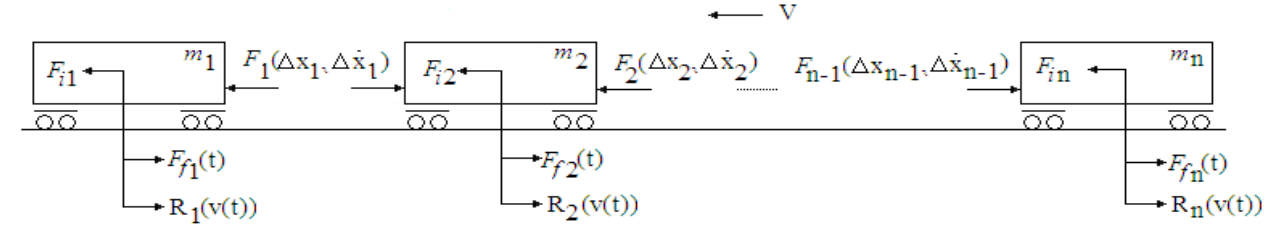

Fig. 1. Forces acting on the train.

The forces acting on each vehicle in the train body represented in the model in Figure 2 are: forces of inertia $F_{i 1}, F_{i 2}, \ldots F_{i n}$; braking forces $F_{f l}(t), F_{f 2}(t), \ldots F_{f n}(t)$; resistance to motion $R_{1}\left(v(t), R_{2}(v(t)), \ldots R_{\mathrm{n}}(v(t))\right.$ and finely forces from buffer and draw-gear devices $F_{1}\left(\Delta x_{1}, \Delta \dot{x}_{1}\right), \ldots F_{n-1}\left(\Delta x_{n-1}, \Delta \dot{x}_{n-1}\right)$.

There were used experimental determinations of the pressure evolution in the brake cylinder for the calculation of the braking force. The maximum braking force developed by each vehicle equal to the wheel-to-rail grip force at the maximum running speed according to basic requirements to be met in designing braking systems of railway vehicles, that is to avoid in normal conditions the locking of the mounted axles during braking [10,15-17].

$$
F_{f, i}(t)=\mu_{a} \cdot m_{i} \cdot g \cdot \frac{p_{c f, i}(t)}{p_{c f \max }}
$$

In the previous relation, $p_{c f i}(t)$ represents the evolution in time of the pressure in the brake cylinder, and $p_{c f m a x}$ is the maximum stabilized pressure.

The resistance to motion of the vehicles in the train body can be determined by:

$$
R_{v, i}=m_{i} \cdot g \cdot r_{v}
$$

Where $r_{v}$ represents the specific resistance to motion, that depends on the type of the vehicle - motor vehicle or dragged vehicle, which in turn may be a vehicle intended for the carriage of passengers or a vehicle intended for the carriage of goods.

Thus, according to [14] the specific resistances to motion can be determined by the following empirical formulas:

- for the vehicles intended for the carriage of passengers:

$$
r_{v}=1.65+\frac{V^{2}(t)}{4000}
$$

- for electrical engines:

$$
r_{v}=\frac{1}{120} \cdot\left[296+7,068 \cdot\left(\frac{V(t)}{10}\right)^{2}\right]
$$

Where $V(t)$ represents the instantaneous speed of the vehicle expressed in $\mathrm{km} / \mathrm{h}$. Due to the fact that relations (4) and (5) are empirical relations specific to each type of vehicle, the relation (3) indicating the value of the resistances to motion for the alignment and elevated plane, the mass of the vehicle $m_{i}$ must be entered in tones, thus the formula can be expressed in $\mathrm{N} / \mathrm{kN}$. 
Applying the laws of mechanics, we obtain $n-1$ nonlinear equations, each describing the movement between two consecutive vehicles. For a vehicle $i$ we will have $[12,14,15]$ :

$$
\begin{aligned}
& \ddot{y}_{i}=\frac{F_{i}\left(y_{i}, \dot{y}_{i}\right)-F_{i+1}\left(y_{i+1}, \dot{y}_{i+1}\right)+F_{f, i+1}(t)+R_{i+1}(v(t))}{m_{i+1}}+ \\
& +\frac{F_{i}\left(y_{i}, \dot{y}_{i}\right)-F_{i-1}\left(y_{i-1}, \dot{y}_{i-1}\right)-F_{f, i}(t)-R_{i}(v(t))}{m_{i}}
\end{aligned}
$$

It is specified that, in order to simplify the above equations, the notation $y_{i}=\Delta x_{i}$ was introduced.

\section{Numerical simulation}

The numerical simulation of the evolution in time of longitudinal dynamic forces, during the period of train braking, between consecutive vehicles, was performed using a program developed in Matlab based on the mechanical model and the formulas presented in the paper, the results being presented in the following Figures.

The simulation was carried out considering two possible situations: a train consisting of ten identical frame-type vehicles and the second case where the train has nine vehicles towed by a locomotive. The simulation scenarios aimed at obtaining the results concerning the longitudinal dynamic forces and their distribution in the train body when the resistances to motion taken into account, respectively are neglected. The locomotive used has six axles, a mass of 120 tons and the towed vehicles are identical passenger coaches whose maximum weight (including the load) is $50 \mathrm{t}$.
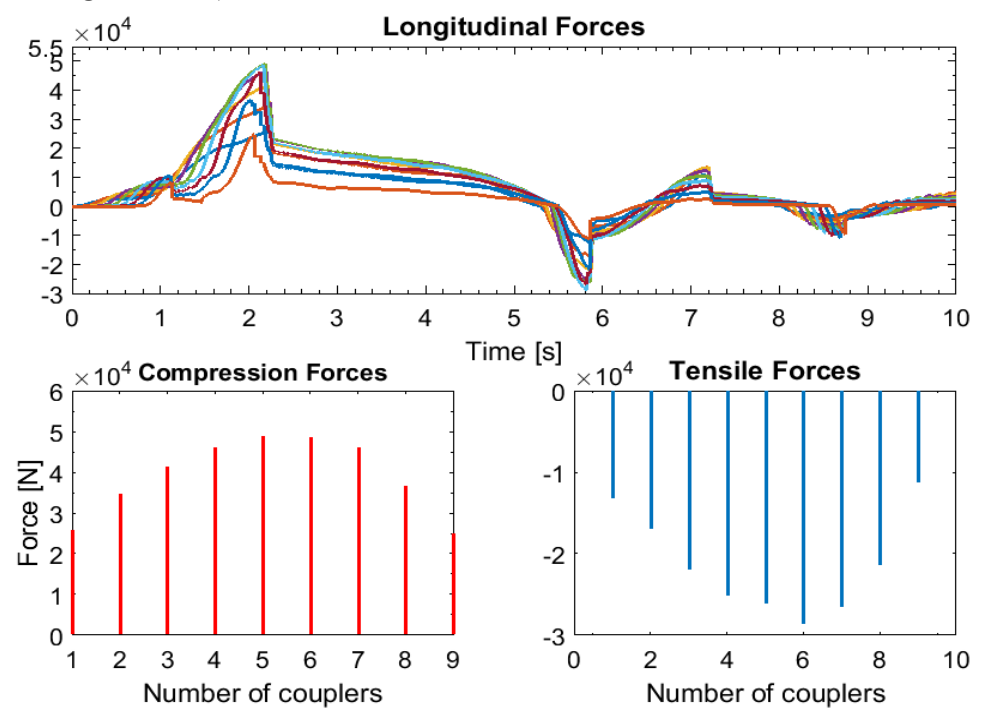

Fig. 2. Longitudinal dynamic forces obtained on the classic train without resistance to motion.

In applying the relation (6) for collision devices (buffers), the constant that depends on the elastic elements has the value $k_{e}=2.8 \cdot 10^{6} \mathrm{~N} / \mathrm{m}$ and the one depending on the friction force $k_{f}=1.410^{6} \mathrm{~N} / \mathrm{m}$. For the traction and coupling devices (mechanical coupling and traction hook), the constant that depends on the elastic elements is $k_{e c}=5.46 \cdot 10^{6} \mathrm{~N} / \mathrm{m}$ and that for the friction force is $k_{f c}=2.43 \cdot 10^{6} \mathrm{~N} / \mathrm{m}$. The braking is achieved from a speed of 150 $\mathrm{km} / \mathrm{h}$, by performing an emergency brake with the achieving of maximum pressure in these 
cylinders. Analyzing the results of the simulation where the resistances to motion are neglected in the case of the frame-type train, it is observed that:

- during the period when the braking forces are developed on each vehicle in the train composition, a compressive force occurs at the level of each collision device, followed by a recoil which develops tensile forces on the binding devices but with a much smaller amplitude than on the compression area;

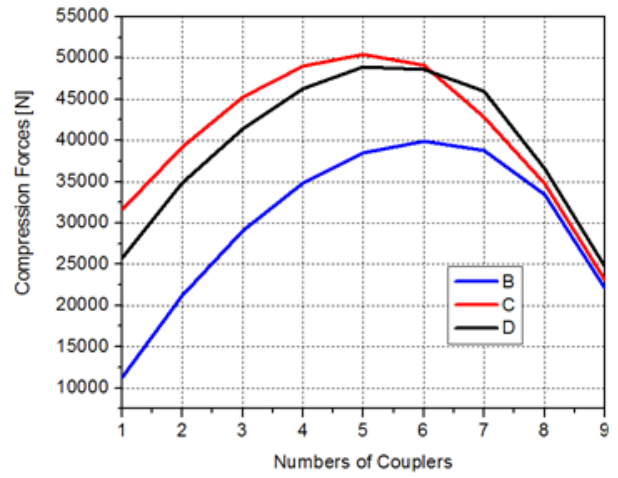

Fig. 3. Distribution of compression forces for all cases. $\mathrm{B}$ - frame train, $\mathrm{C}$ - train with resistance, D - train without resistance

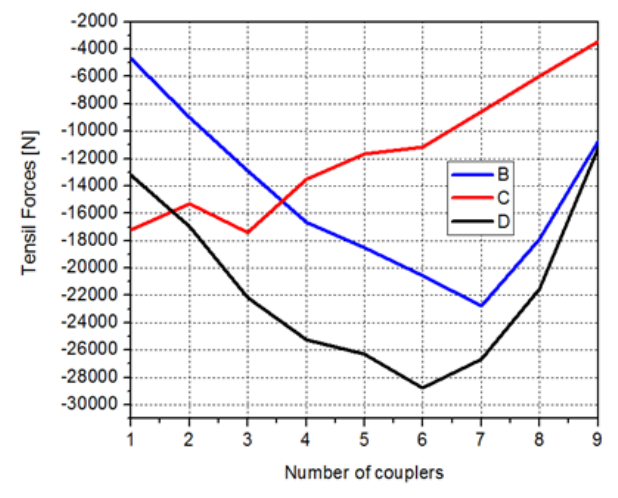

Fig. 4. Distribution of tensile forces for all cases. B - Frame train, $\mathrm{C}$ - train with resistance, $\mathrm{D}$ - train without resistance

- regarding the forces distribution, it can be seen from the histograms shown in Figure 3 that the highest compression force is recorded on the buffer between vehicles 6 and 7, that is in the second half of the train, being very close to Karvatki's theory stating that maximal forces are developing halfway through the train;

- tensile forces have the highest values in the second half too, showing a linear increase to the maximum value (coupler number 8 according to the histogram in Figure 4), then they begin to decrease to the end of the train.

To see the effect on a classic train composed of a locomotive and wagons, the simulation was carried out both with and without resistance to motion. Comparing the results obtained for the situation without resistances to motion, it is observed that in this case there are notable differences between the results of the simulations; the level of the longitudinal dynamic forces has significantly increased, a first explanation being that a concentrated mass, equivalent of three wagons was introduced into one of the train heads Comparing the results obtained between the frame and the train, without using the resistances to motion, it can be observed that the distribution rate of the compression forces and the tensile forces is preserved, but the maximum obtained values significantly increased by an average value of approximately $38 \%$ (Figure 3 ) while tensile forces doubled or tripled for the couplers at the beginning of the train, the differences between them decreasing considerably for the couplers at the end of the train (Figure 4). When the resistances to motion are introduced, significant changes occur in the results obtained for the classic train. It can be seen that the distribution of the tensile forces has the tendency to order Gaussian in relation to the train length (Figure 3-C), respecting this time the hypothesis that the maximum value is recorded on the group of buffers located in the middle of the train. The tensile forces have a completely different distribution compared to the other situations (Figure 4-C), having the maximum values on the couplings at the beginning of the train, following an almost linear decrease towards the end of the train. It can therefore be said that this situation is possible because the specific resistance of the locomotive is up to 10 times higher than that of a regular passenger coach leading to much 
higher recoils than when the calculations are performed without holding account of these resistances to motion.

\section{Conclusions}

During train braking, longitudinal dynamic forces develop in buffer and draw-gear devices. These are due to the differences between the braking forces developed on each train vehicle as a result of successive entry into action of the individual braking installations. Longitudinal dynamic forces overload the load-bearing structure of the vehicle and create the possibility of some shocks in the train body that can affect the comfort of the passengers and in some situations can lead to train break even with the damage of traffic safety.

The paper highlights the differences that appear from the simulations between the value and the distribution of the longitudinal forces when we use or not the resistance to motion in these programs. In reality, the resistances to motion depend on many parameters and sometimes there is a tendency to ignore them into the simulation programs in order to simplify the results interpretation. Following this analysis it can be concluded that:

- when simulations are carried out for identical frames or vehicles, in order to highlight the modifications made by a particular parameter or simply for estimated calculations, the use of resistances to motion may be given up;

- in the case of long trains (locomotive and wagons), high-speed brakes or load-bearing for each coupling coupler, the introduction into the simulation programs of the forces given by the resistances to motion becomes a necessity which, although makes harder the program running, can deliver results much closer to reality.

\section{References}

1. M. Spiryagin, Q. Wu \& C. Cole: Veh. Syst. Dyn. 55, (2017)

2. C. Cole Longitudinal train dynamics, chapter 9 in Handbook of Railway Vehicle Dynamics (Ed. Taylor \& Francis Grup, (2006)

3. P. Belforte, F. Cheli, G. Diana, S. Melzi, Veh. Syst. Dyn., 19 (2008)

4. A. Nasr, S. Mohammadi, Proc. ImechE, J. Rail and Rapid Transit, 224, 11 (2010)

5. L. Pugi, D. Fioravanti \& L. Rindi, 12th IFToMM World Congress, (2007)

6. L. Pugi, M. Malavezzi, S. Papini, G. Vellori, J. of Modern Trans. 21, 247-257 (2013)

7. L. Pugi, M. Malavezzi, B. Allotta, L. Banchi, P. Presciani, Proc. IMechE 218 Part F: J. Rail and Rapid Transit (2004)

8. J. Wang, H. A. Rakha, Transportation Research, C, Emerging Tech., 86 (2017)

9. Q. Wu, C. Cole, S. Luo, M. Spiryagin, Veh. Syst. Dyn, 52, 6, 733-758, (2014)

10. C. Cruceanu, R. Oprea, M. Spiroiu, C. Crăciun, S. Arsene, WSEAS Int. Conf. Proceedings. Recent Advances in Computer Engineering, 13, 531-536, (2009)

11. C. Cruceanu, M. Dumitriu, C. Crăciun, MATEC Web of Conf., 112 (2017)

12. C. Crăciun, T. Mazilu, Ann. Fac. Eng. Hunedoara - Int. J. Eng., 12, 8 (2014)

13. I. Sebeşan, C. Crăciun, A.M. Mitu, UPB. Sci. Bull. Series D, 76,76-88 (2014)

14. C. Crăciun, M. Dumitriu, Appl. Mech. Mater., 809-810, 6 (2015)

15. C. Cruceanu, Train Braking, in Reliability and Safety in Railway 29-74, InTech (2012)

16. C. Crăciun, M. Dumitriu, C. Cruceanu, MATEC Web of Conf., 112, (2017) 
17. C. Cruceanu, C.I. Crăciun, I.C. Cruceanu, Rail Transport-Systems Approch, 87, 3-33, Springer International (2017) 